\title{
MOBILITY LOAD BALANCING BASED ADAPTIVE HANDOVER IN DOWNLINK LTE SELF-ORGANIZING NETWORKS
}

\author{
Hana Jouini ${ }^{1}$, Mohamed Escheikh ${ }^{1}$, Kamel Barkaoui $^{2}$ and Tahar Ezzedine ${ }^{1}$ \\ ${ }^{1}$ University of Tunis El Manar, Enit, Sys'Com , 1002 Tunis, Tunisia \\ ${ }^{2}$ Cedric-Cnam : 2 Rue Conté 75003 Paris, France
}

\begin{abstract}
This article investigates mobility load balancing (MLB) algorithm implementation through network simulator (ns-3) in long term evolution (LTE) systems employing orthogonal frequency division multiple access (OFDMA) for downlink (DL) data transmission. MLB is introduced by the third generation partnership project (3GPP) as a key target of LTE self-organizing networks (SONs) [1]. Our contribution is twofold. First, we implemented elementary procedures (EPs) related to load management (LM) function of the X2-application protocol (X2AP) as specified in TS 136.423 [2]. We particularly focused on EPs 'Resource Status Reporting Initiation Procedure' and 'Resource Status Reporting Procedure'. Second, we implemented a MLB based adaptive handover (HO) algorithm enabling to configure adaptively $\mathrm{HO}$ hysteresis threshold for each neighbouring cell, of an overloaded cell, according to its current load information. Numerical results show how, through suitable simulation scenarios, MLB enables enhancing network performance in terms of overall throughput, packet loss ratio (PLR) and fairness without incurring HO overhead.
\end{abstract}

\section{KEYWORDS}

LTE, load management, X2AP, elementary procedure, mobility load balancing

\section{INTRODUCTION}

In recent years, wireless cellular networks' operators have experienced a spectacular growth in mobile data traffic. With the surging traffic demand, wireless cellular networks are becoming significantly more complex, causing higher operational costs. In order to reduce these costs while optimizing network efficiency and service quality, the SON concept had been introduced in LTE systems. SON concept aims to reduce manual operations by integrating self-optimizing, selfhealing and self-configuration features. MLB is one of the most important functionalities that belong to self-optimizing SON techniques.

In wireless cellular networks, loads in different cells are frequently unequal. This leads to critical situation where hot spot cells (i.e. overloaded cells) suffer from a high call blocking rate (CBR) and/or call dropping rate (CDR). In contrast, a large part of resources in low-loaded cells remains in idle state causing resource wastes. Therefore, load imbalance between neighbouring cells seriously deteriorates network performance. In this regard MLB solutions are deployed to circumvent this problem and to enhance fairness, availability, scalability, user-experience and network utilization.

DOI: 10.5121/ijwmn.2016.8406 
In literature several load balancing methods devoted to cellular networks have been proposed. Authors in [3] formulate a multi-objective optimization problem using utility function jointly optimizing load balancing (LB) index and network average load for users with quality of service (QoS) requirements. They conclude that better values of LB index and network average load can be achieved when compared with other conventional methods. In $[4,5]$, authors investigate conflicting and stand-alone SON functions. They particularly focused on two SON functions namely MLB and handover optimization (HOO). In this direction also and in order to optimize the same parameter values having conflicting goals at the same network element (NE), 3GPP proposes an additional entity, usually called coordinator [21]. Alternatively, to reach the above purpose, authors in [4] propose a unified self-management mechanism for MLB and HOO based on fuzzy logic and reinforcement learning techniques. The objective behind is to reduce SON coordination entity's complexity. Authors in [5] propose an optimization technique of both MLB and $\mathrm{HOO}$ functions based on a weighted co-satisfaction factor (CSF). Another practical solution for MLB is proposed in [6] and is based on HO margins' auto-tuning of each base station according to its load and the load of its neighbouring cells. Authors highlight through simulation analysis MLB impact on both call admission rate and user throughput. Z. Huang et al. propose in [7] a multi-traffic load balance algorithm (MTLB) to promote an efficient load balance. Yang et al. propose in [8] an auto-configuration technique of handover hysteresis threshold according to the load information of the current overloaded cell and its neighbours. The proposed technique triggers the MLB procedure according to the load condition of a cell and significantly reduces $\mathrm{CBR}$, HO dropping/blocking rate and ping-pong $\mathrm{HO}$ rate. However, the paper neglects the impact of the proposed mechanism on some key performance metrics such as the global throughput in the network and the LB index. The proposed model in the above paper is implemented using a formal description technique based on a specification and a description language (SDL). Thereby algorithm efficiency in [8] needs to be improved for more realistic conditions and in this regard a more realistic implementation with respect to the 3GPP specifications could be established. In this paper we propose a MLB algorithm implementation based on discrete-event network simulator ns-3 [17]. To that end two implementation steps are followed. In a first step two LM EPs of the of X2AP protocol namely 'Resource Status Reporting Initiation Procedure' and 'Resource Status Reporting Procedure' are implemented as specified in TS 136.423 [2]. We exploit next these EPs to implement in a second step the MLB algorithm proposed in [8] based on adjusting adaptively $\mathrm{HO}$ hysteresis threshold according to cell load information. We specifically focus on A3 HO triggering event based on measurements of the reference signal received power (RSRP) [9]. Numerical results show how MLB enhances network performance without incurring significant overhead. We particularly show for DL how performance metrics such as global network throughput, PLR, number of successful HO and LB fairness index evolve for different user equipment (UE) densities.

The rest of this paper is organized as follows. Section II presents X2AP HO and LM signalling functions in $3 \mathrm{GPP}$. We succinctly recall in section III some definitions related to MLB based adaptive handover for LTE Systems. In section IV we provide a simulation model description of the LTE system adopted in this paper. We propose in section V the X2AP EPs extension implemented in ns-3 before detailing in section VI, based on X2AP functionalities, a MLB algorithm implementation for LTE systems in DL. Finally, simulation results are presented in section VII for different KPIs metrics and concluding remarks are provided in section VIII. 
International Journal of Wireless \& Mobile Networks (IJWMN) Vol. 8, No. 4, August 2016

\section{HO AND MLB Signalling Functions OF X2AP IN 3GPP SPECIFICATIONS}

In LTE architecture, the core network (CN) includes mobility management entity (MME), serving gateway (SGW) and packet data network gateway (PDN-GW), whereas E-UTRAN includes eNodeBs (eNBs) [10]. The X2 interface is defined between two neighbouring eNBs at two planes (Figure 1) [11]:

- X2-UP (User-plane): is the user-plane interface between two neighbouring eNBs. X2-UP protocol tunnels end-user packets between eNBs. The transport network layer is built on IP transport, and GTP-U is used on top of the UDP to carry the user-plane PDUs.

- X2-CP (Control-plane): is the control plane interface between two neighbouring eNBs. $\mathrm{X} 2-\mathrm{CP}$ has stream control transmission protocol (SCTP) as transport layer protocol and $\mathrm{X} 2 \mathrm{AP}$ as application layer signalling protocol.

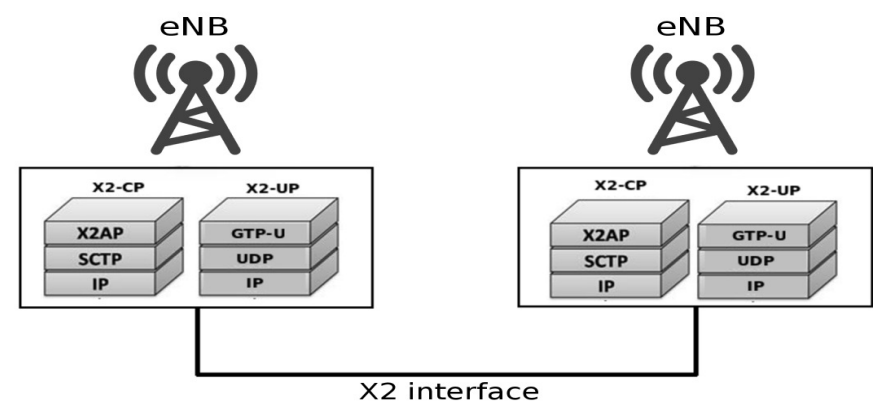

Figure 1. Protocol stack of the X2 interface

X2AP supports radio network layer signalling functions of the control plane between eNBs. 3 GPP defines X2AP as a collection of EPs [2]. A X2AP EP is a unit of interaction between two eNBs. Since X2AP is responsible of signalling between two neighbouring eNBs, it ensures two major SON functions namely $\mathrm{HO}$ signalling functions (i.e. mobility management (MM), mobility robustness optimization (MRO), mobility parameters management (MPM) and load information exchanges function (i.e. LM)). The HO enables one eNB to hand over an user equipment (UE) to another eNB and requires the transfer of useful information to maintain the LTE radio access network (RAN) services at the new eNB. The LM function allows to exchange traffic load and its related signalling between neighbouring eNBs. In what follows we give more details about HO and LM functions.

\subsection{HO Functions}

In LTE the HO procedure is based on UE's measurements; A serving eNB sends a connection reconfiguration message through the radio resource control (RRC) layer to inform an UE of its related measurement configuration. If the UE detects any event related to measurement configuration, a measurement event is triggered and the serving eNB is informed. The decision of triggering a $\mathrm{HO}$ is based on the detection of a special event. 3GPP specifies several reporting 
events to trigger HO [9] depending on the measurements' values. The serving eNB should indicate to UEs the event to use in the connection reconfiguration message. In order to trigger a HO within LTE several kind of events may be adopted.

Our choice in this paper is directed toward A3 event, which might be triggered if a neighbouring cell measurement becomes offset better than the serving cell. The entering condition to be satisfied for A3 event can be simplified as follows:

$M n>M s+H y s+O f f+$ Freq + SOff

where $M s$ is the UE measurement corresponding to the serving cell and $M n$ is the UE measurement corresponding to the neighbouring cells. As part of the reporting configuration, the eNB can tell the UEs to measure either their cells RSRP, or their reference signal received quality (RSRQ) [12]. Hys is a hysteresis parameter for measurement reporting. If the UE sends a measurement report to its serving cell, then Hys prevents any more reports until the signal level changes by $2 \cdot$ Hys (Figure 2). Similarly, Off is a hysteresis parameter for HOs. If the measurement report triggers $\mathrm{HO}$, then Off prevents the UE from moving back to the original cell until the signal level changes by $2 \cdot$ Off . Freq (resp. SOff) is the difference between frequency specific offsets (resp. cell specific-offsets) of the serving and neighbouring cells [9]. The time to trigger (TTT) parameter is the time during which the condition of triggering A3 event needs to be maintained in order to trigger a measurement report (Figure 2).

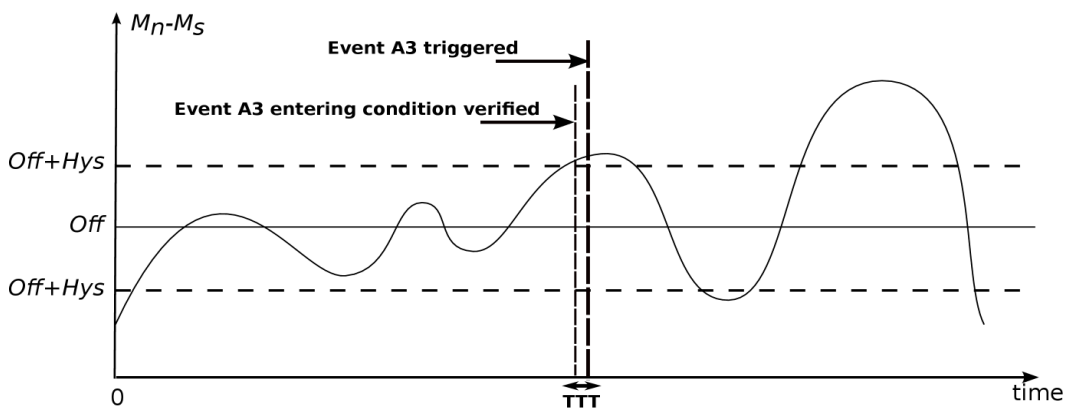

Figure 2. Measurement event A3 with Freq $=$ SOff $=0$

If a measurement report is sent to the serving cell with a triggered $\mathrm{A} 3$ event, this latter may trigger a $\mathrm{HO}$ procedure by starting a MM function with the neighbouring cell. In what follows we describe the MM function of the X2AP protocol.

\subsubsection{Function}

The MM function covers four EPs [2]:

- Handover Preparation EP: during this procedure the serving cell initiates the HO with the HANDOVER REQUEST message and includes necessary information for neighbouring cell preparation to an incoming HO. The neighbouring cell responds back to the serving cell with a HO REQUEST ACKNOWLEDGE message (successful operation) or a HANDOVER PREPARATION FAILURE message (unsuccessful operation); 
International Journal of Wireless \& Mobile Networks (IJWMN) Vol. 8, No. 4, August 2016

- SN Status Transfer EP: along this procedure the SN Status Transfer is sent from the serving cell to its neighbours to transmit uplink (UL) and DL packet data convergence protocol sequence number (PDCP SN) and hyper frame number (HFN) receiver status and transmitter status from the source eNB to the target eNB during an X2 handover. This is achieved for each evolved radio access bearer (E-RAB) for which PDCP SN and HFN status preservation applies [2];

- UE Context Release EP: By sending an UE CONTEXT RELEASE message, the target eNB informs the source eNB of HO success and a release of resources by the eNB source is triggered. The target eNB sends this message after receiving the PATH SWITCH ACKNOWLEDGE message from the MME. Upon reception of the UE CONTEXT RELEASE message, the source eNB can release data and C-plane related resources associated to the UE context. Therefore, any ongoing data forwarding may continue;

- Handover Cancel EP: is used by a source eNB to cancel an ongoing HO preparation or an already prepared HO.

\subsubsection{MPM Function}

MPM function covers one EP: the mobility settings change (MSC) EP. This latter enables an eNB to negotiate the $\mathrm{HO}$ trigger settings with a peer eNB controlling neighbouring cells [2].

\subsubsection{MRO Function}

MRO function detects and corrects automatically errors in the mobility configuration. It covers two EPs:

- Radio link failure (RLF) indication EP [2]: The purpose of this EP is to transfer information regarding RRC re-establishment attempts or received RLF reports, between eNBs;

- Handover report EP: This EP enables to transfer mobility related information between eNBs.

\subsection{LM Function}

In order to detect a load imbalance between eNBs in the network, it is necessary to compare cells' load information and exchange them between neighbouring eNBs [13].

Three separate EPs are used to exchange load information via the X2 interface:

- Load Indication EP: The load indication procedure is used over the X2 interface for load and interference management information exchange. An eNB initiates this EP by sending LOAD INFORMATION message to a neighbouring eNB;

- Resource Status Reporting Initiation EP and Resource Status Reporting EP (Figure 3): These EPs are used to initiate and report load measurements result between eNBs. A source eNB sends a RESOURCE STATUS REQUEST message to a target eNB. If this latter is able to provide requested resource status information, it responds with the 
RESOURCE STATUS RESPONSE message and then Resource Status Reporting EP could be initiated. The target eNB will report measurement results in RESOURCE STATUS UPDATE message.

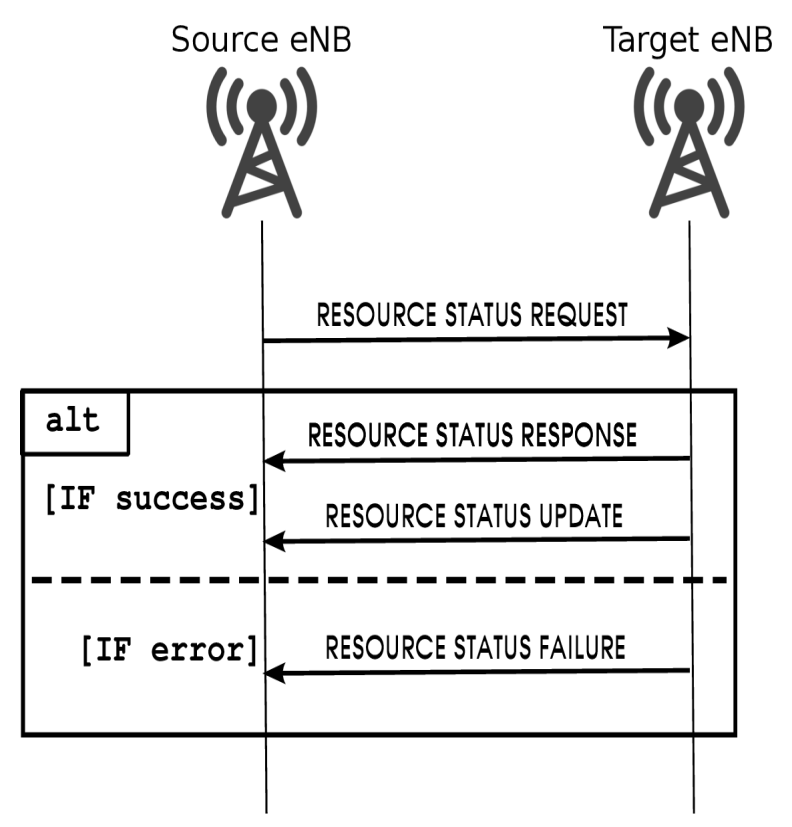

Figure 3. Resource Status Reporting EP

\section{MLB BASED ADAPTIVE HO IN DL LTE SELF-ORGANIZING NETWORKS}

To improve the overall system capacity 3GPP defines a self-optimizing function namely MLB. MLB enables cells suffering from congestion to transfer extra-load to other neighbouring cells willing to cooperate toward their spare resources [14]. The MLB is governed by load reporting mechanisms activated between eNBs (over the X2 interface) to exchange information about load level and available capacity. One solution to introduce MLB in a LTE system is to steer extratraffic toward neighbouring cells by optimizing dynamically the cell $\mathrm{HO}$ parameters, such as hysteresis, upon detection of an imbalance [13]. In fact, SON techniques allow to automatically adjust mobility configuration based on several factors such as received load information.

In order to describe MLB principle let's consider event A3 (Figure 2) described above and assume that it is triggered between an overloaded cell and its neighbour at time T1. The decreasing of the Hys value fosters the triggering of A3 event $(T 2<T 1)$ and consequently facilitates the HO triggering from the overloaded cell to its neighbouring cell (Figure 4). By adjusting Hys value, the hot spot cell forces UEs to hand over to its neighbouring cell. Then MLB based adaptive $\mathrm{HO}$ may be defined as an explicit triggering of a forced $\mathrm{HO}$ for load balancing purposes. To apply this mechanism, each cell should be aware of its own load conditions as well as the load of its neighbours. 


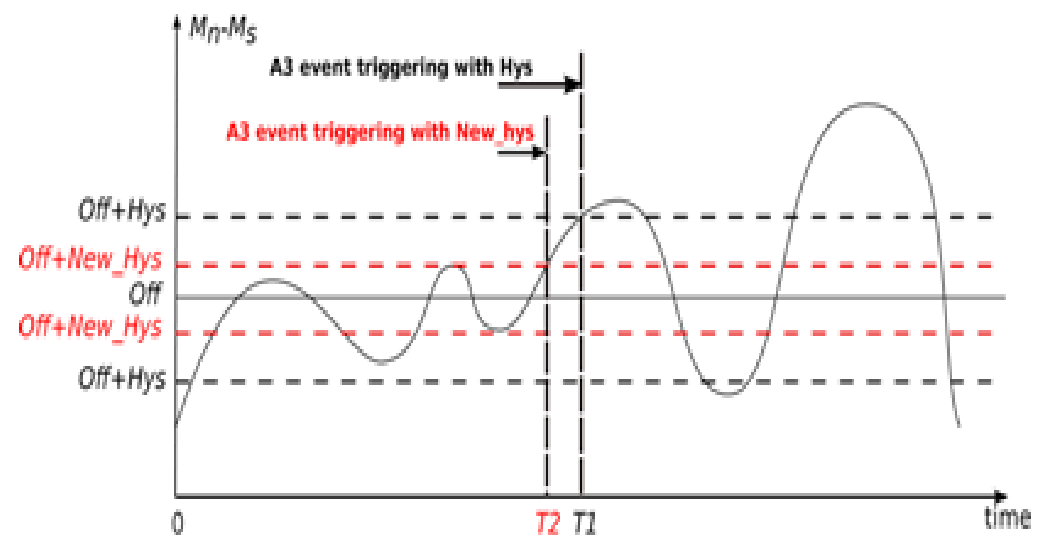

Figure 4. Sample of dynamic hysteresis adaptation effect on HO triggering when using A3 event with Freq $=S O f f=0$ (To simplify the figure, TTT is not represented)

This enables respectively to detect an overload situation and to select the best neighbouring cell to hand over UEs. For that purpose, eNBs periodically monitor their own load conditions and exchange load information over the X2 interface (Figure 3). The RESOURCE STATUS UPDATE message includes a special attribute called composite available capacity (CAC) [2]. CAC represents the overall available resource level that can be offered for MLB purposes in either DL (CACD) or UL (CACU). CAC can be represented as a data structure including two information elements (IE) [2]:

- Cell Capacity Class Value IE: classifies the cell capacity with regards to the other cells. The Cell Capacity Class Value IE indicates resources that may be reserved for data traffic;

- Capacity Value IE: Indicates the ratio between the amount of available resources and the amount of total E-UTRAN resources. The Capacity Value IE is measured and reported so that suitable E-UTRAN resources of the existing services are reserved. The Capacity Value IE can be weighted according to the ratio of cell capacity class values.

In this paper we turn our interest only to CACD which is not supported by the eNB model in given in current version of ns-3 (i.e. ns-3.24) [17]. In this regard we will define and develop the calculation of CACD. In the next section we will describe the system model and explain basic set notation we used for the formulation of the treated problem.

\section{SYSTEM MODEL}

We consider in this paper a scenario of a multi-cell LTE network (Figure 5) where each eNB consists of three cells. Let $C$ denotes the set of all cells in the LTE network. $C_{i}$ represents the cell $i(i \in[1 . . C])$. 


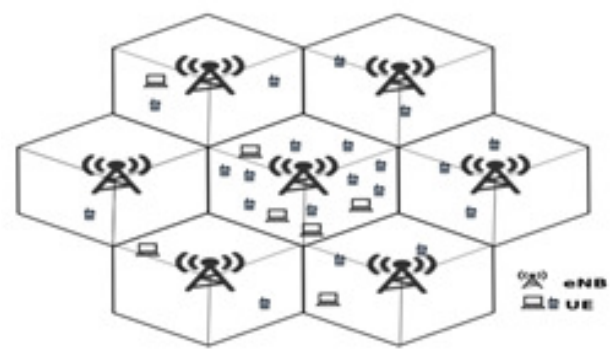

Figure 5. Network model

$C_{s}$ is used to indicate the serving cell. $C_{i, j}$ denotes a neighbouring cell $C_{j}$ of a cell $C_{i}(j \in[1 . . C]$ ). $C_{i, S j}$ indicates the set of all neighbours $S j$ of $C_{i}$. The amount of resource blocks (RBs) quantifying time-frequency resources of $C_{i}$ is denoted $R_{i}$ (We assume that all cells have the same amount of RBs). $U$ is the set of all UEs scheduled in the network (we assume that $U$ is constant along the simulation duration), and $U_{i}(t)$ is the set of UEs scheduled in $C_{i}$ at time $t$ and $U_{i, k}(t)$ indicates an UE $k$ scheduled in $C_{i}$ at time $t . R_{i, k}(t)$ is the amount of consumed RBs by $U_{i, k}(t)$ at time $t . H Y S_{i, j}$ is the hysteresis value of $\mathrm{HO}$ from $C_{i}$ to its neighbouring cell $C_{i, j}$. The load $\rho_{i}(t)$ of $C_{i}$ at time $t$ can be calculated as follows:

$\rho_{i}(t)=\frac{\sum_{k \in U_{i}(t)} R_{i, k}(t)}{R_{i}}$

Then CACD of $C_{i}$ at time $t$ (i.e. $\left.C A C D_{i}(t)\right)$ could be defined as follows [15]:

$C A C D_{i}(t)=100 \cdot\left(1-\rho_{i}\right)$

To measure network performance, we use the following metrics:

- network average load in DL $\zeta$ :

$\rho(t)$ is the network load at time $t$ :

$\rho(t)=\frac{\sum_{i=C} \rho_{i}(t)}{C}$

Let $T$ denotes the simulation duration and $p$ the epoch duration. Then $\zeta$ can be calculated as follows:

$$
\zeta=\frac{\sum_{i \in C} \sum_{t \in T} \rho_{i}(t)}{C \cdot\left(\frac{T}{p}\right)}
$$


- Jain's fairness index [16]:

$F(t)$ is the Jain's fairness index at time $t$ :

$$
F(t)=\frac{\left(\sum_{i \in C} \rho_{i}(t)\right)^{2}}{C \cdot \sum_{i \in C} \rho_{i}^{2}(t)} \in[0 . .1]
$$

then the Jain's fairness index $F$ can be calculated as follows:

$$
F=\frac{\left(\sum_{i \in C} \sum_{i \in T} \rho_{i}(t)\right)^{2}}{C \cdot \sum_{i \in C}\left(\sum_{i \in T} \rho_{i}(t)\right)^{2}} \in[0 . .1]
$$

- Packet loss ratio (PLR) in DL: is defined as the difference between the number of transmitted packets and the number of received packets.

- Number of successful HO: Since ns-3 in its current version (i.e. ns-3.24) [17] doesn't support the HO Failure procedure, the number of successful HO is indeed the number of triggering (or the triggering frequency) of a $\mathrm{HO}$ procedure. The following section discusses the proposed X2AP EPs extension proposed in this paper for ns-3.

\section{The Proposed X2AP EPs EXTENSION}

Ns-3 [17] is an open-source discrete event network simulator, developed using C++ programming language. Ns-3 supports the simulation of 3GPP LTE cellular networks. Baldo et al. propose in [18] a simulation model for LTE HO scenarios using ns-3. In order to integrate SON functionalities, the proposed model implements X2 interface supporting RESOURCE STATUS UPDATE message. Such implementation offers only a prototype of this message and details regarding associated variables are not specified. In this paper we implement new added methods illustrated in Figure 6. This is achieved through two implementation steps. The first one concerns RESOURCE STATUS REQUEST and RESOURCE STATUS RESPONSE messages (i.e. Resource Status Reporting Initiation EP), which are necessary for the initiation of a RESOURCE STATUS UPDATE message (RESOURCE STATUS FAILURE message is not considered in this paper). Whereas the second focuses on RESOURCE STATUS UPDATE message (i.e. Resource Status Reporting EP). The added methods are detailed as follows:

- LteEnbRrc::DoSendResourceStatusRequest(): An overloaded cell executes this function to interrogate neighbouring cells about their load conditions.

- LteEnbRrc::DoRecvResourceStatusRequest(): This function is executed when a cell receives a RESOURCE STATUS REQUEST message.

- LteEnbRrc::DoSendResourceStatusResponse(): A cell that receives a RESOURCE STATUS REQUEST message will execute this method to send an acknowledgement to its neighbour.

- LteEnbRrc::DoRecvResourceStatusResponse(): This function is executed when a cell receives a RESOURCE STATUS RESPONSE message. 
- LteEnbRrc::DoSendResourceStatusUpdate(): A cell calculates its CACD and executes this function to send it to a neighbouring cell.

- LteEnbRrc::DoRecvResourceStatusUpdate(): This function is executed when a cell receives a RESOURCE STATUS UPDATE message.

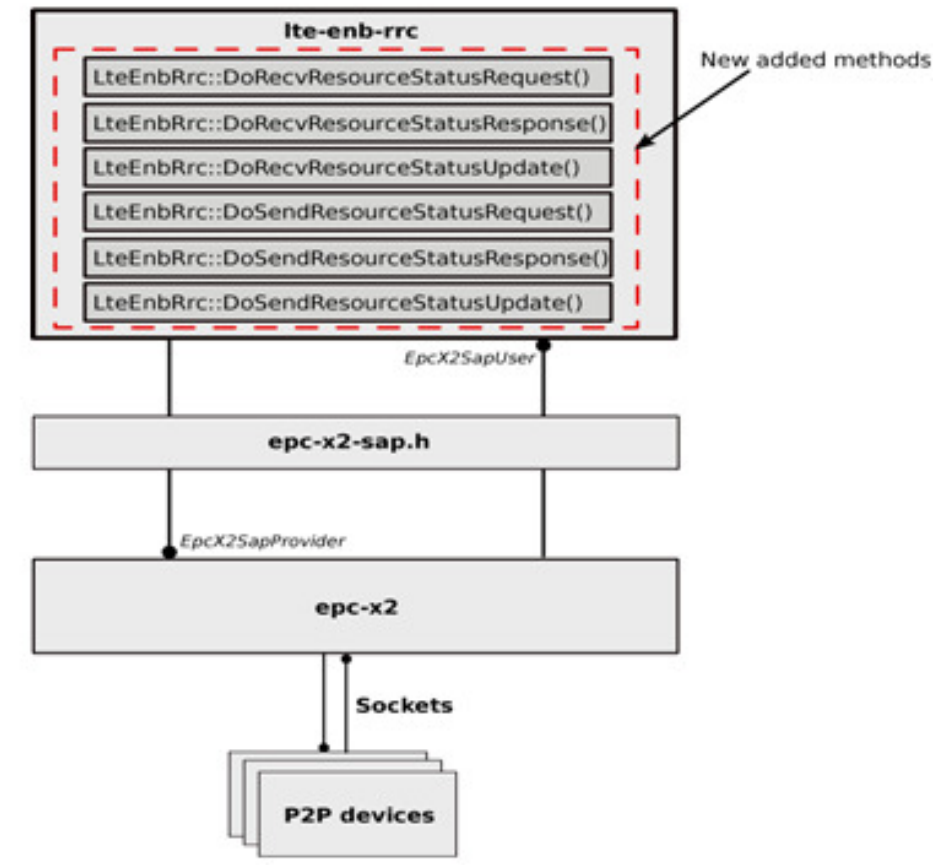

Figure 6. Implementation model of X2 entity and Service Access Points (SAP)

\section{MLB Proposed Algorithm}

In our proposed MLB algorithm we assume $C_{i}$ is overloaded if Cond. 1 is satisfied (Table 1). Indeed if $C A C D_{i}$ is less than a given threshold $\operatorname{ThPre}_{L B}$, then $C_{i}$ is considered as a hot spot (i.e. overloaded) cell and it enables the MLB algorithm. Now if Cond. 2 is satisfied, this means that $C_{i}$ is low-loaded enough to disable the MLB algorithm.

Table 1: Conditions enabling/disabling MLB algorithm

\begin{tabular}{|c|c|}
\hline Condition & Action \\
\hline $\begin{array}{l}\text { Cond. 1: } \\
C A C D_{i} \leq \text { ThPre }_{L B}\end{array}$ & $\begin{array}{l}C_{i} \text { falls into the heavy load condition and the MLB } \\
\text { algorithm is enabled. }\end{array}$ \\
\hline $\begin{array}{l}\text { Cond. 2: } \\
\text { CACD }_{i} \geq \text { ThPost }_{L B}\end{array}$ & $\begin{array}{l}C_{i} \text { falls into the low load condition and the MLB } \\
\text { algorithm is disabled. }\end{array}$ \\
\hline
\end{tabular}

The proposed MLB algorithm is based on the adaptive modification of actual hysteresis value, between a serving hot spot cell $C_{s}$ and its neighbouring cells $C_{s, S j}$, according to the neighbouring 
International Journal of Wireless \& Mobile Networks (IJWMN) Vol. 8, No. 4, August 2016

cells' load. Enabling the MLB algorithm means that $C_{s}$ is allowed to send RESOURCE STATUS REQUEST messages to $C_{s, S j}$ (Figure 7). $C_{s}$ will update $H Y S_{s, j}\left(j \in S j\right.$ ) value only if $C_{s, j}$ answers with a RESOURCE STATUS RESPONSE message (Figure 3).

If a status error occurs, then $C_{s, j}$ sends a RESOURCE STATUS FAILURE message and $C_{s}$ will not update the $H Y S_{s, j}$ value (Figure 7).

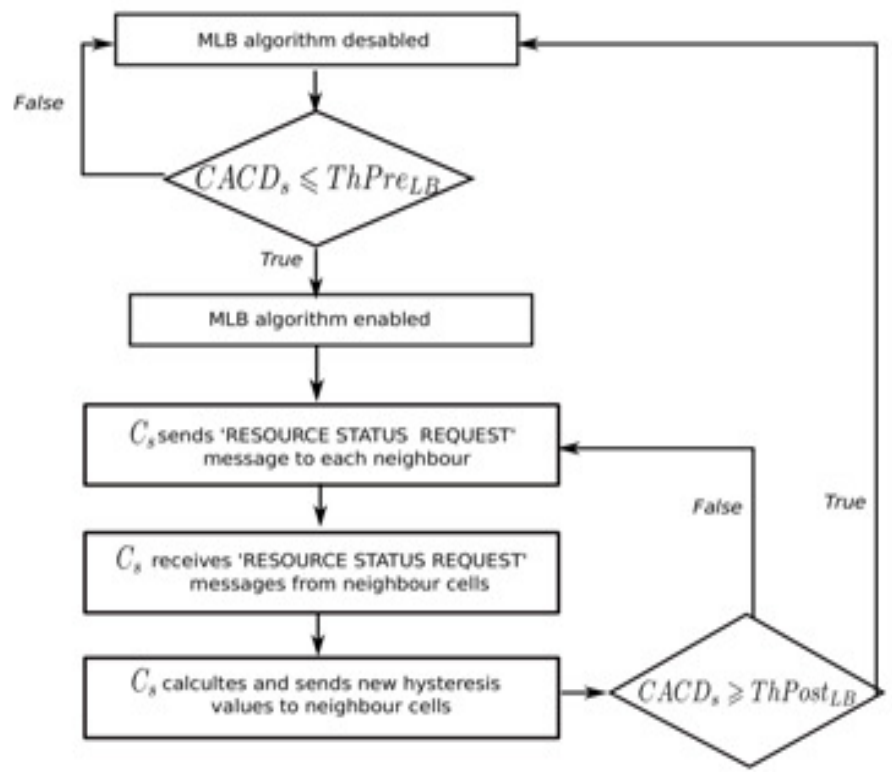

Figure 7. Sequence diagram of the proposed MLB algorithm

The new hysteresis value between $C_{s}$ and $C_{s, j}$ (i.e. Newhys $s_{s, j}$ ) is adjusted as follows:

$$
\text { Newhys } s_{s, j}=\alpha_{s, j} \cdot h y s_{s, j} \quad, \quad j \in S j
$$

Where $\alpha_{s, j}$ is a weighting factor in [0..1] calculated as follows (Figure 8):

$$
\alpha_{s, j}=\left\{\begin{array}{ccc}
1 & \text { if } & \text { CACD }_{j}<\text { ThAvail }_{L B} \\
1-\frac{\text { ThAvail }_{L B}-\text { CACD }_{j}}{\text { ThAvail }_{L B}-\text { ThPost }_{L B}} & \text { if } & \text { ThAvail }_{L B} \leq \text { CACD }_{j}<\text { ThPost }_{L B} \\
0 & \text { if } & \text { CACD }_{j} \geq \text { ThPost }_{L B}
\end{array}\right.
$$

Figure 8 represents the $\alpha_{s, j}$ evolution with respect to $C A C D_{j}$. 


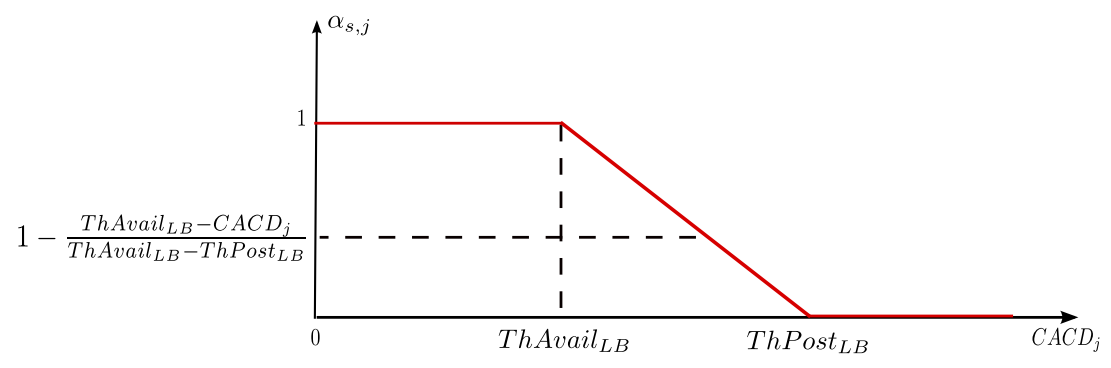

Figure 8. Graphical representation of $\alpha_{s, j}$ calculation

\section{Performance Evaluation}

\subsection{Simulation Setup}

Table 2 summarizes the main parameters of the simulation scenario where a multi-cell LTE network composed of 7 eNBs ( 21 cells) in hexagonal layout (Figure 5) is considered.

Distance between eNBs is assumed equals to 500 meters. In order to create an overloaded situation, we initially attached all UEs to one cell $\left(C_{l}\right.$ of the central eNB). The mobility pattern considered is the Random Waypoint model $[19,20]$. In such model nodes move independently to a randomly chosen destination with a randomly selected velocity. This allows to emulate a real life situation in a reasonable way. The number of UEs in the simulation scenarios can be changed by choosing a proper UEs density $\lambda$. We conducted a simulation campaign with different number of UEs $(U \in\{25,50,75,100,150\})$ in order to investigate load impact on performance of the proposed MLB algorithm.

Table 2. Simulation parameters

\begin{tabular}{|l|l|}
\hline Parameter & Value \\
\hline Cellular layout & $7 \mathrm{eNBs}(21$ cells) in hexagonal layout \\
\hline Inter-eNBs distance & $500 \mathrm{~m}$ \\
\hline Cell Tx power & $46 \mathrm{dbm}$ \\
\hline Path loss model & $L=128.1+37.6+\log (R)$ \\
\hline Channel fading & Typical urban \\
\hline Carrier frequency & $2 \mathrm{Ghz}$ \\
\hline System bandwidth & $5 \mathrm{Mhz}(25 \mathrm{RBs})$ \\
\hline Traffic & Only control messages, no data traffic \\
\hline Error model & None \\
\hline UE distribution & $U \in\{50,75,100,125,150\}$ attached all to $C_{l}$ of the \\
& central eNB \\
\hline UE movement pattern & $30 \mathrm{dbm}$ \\
\hline UE measurement reporting interval & $50 \mathrm{~ms}$ \\
\hline Simulation duration & $50 \mathrm{~s}$ \\
\hline Time To Trigger (TTT) & $256 \mathrm{~ms}$ \\
\hline Hysteresis default value & $3 \mathrm{db}$ \\
\hline Hysteresis margin with MLB & {$[0 . .3 \mathrm{db}]$} \\
\hline
\end{tabular}




\begin{tabular}{|l|l|}
\hline ThPreLB & 0.3 \\
\hline ThAvailLB & 0.4 \\
\hline ThPostLB & 0.6 \\
\hline
\end{tabular}

\subsection{Simulation Results}

We investigate in this section numerical results related to MLB algorithm implementation. Figure 9 exhibits the LTE network global throughput in downlink. As observed, the MLB impact on network performance depends closely on UEs density. We distinguish two kinds of LTE network global throughput behaviour with respect to UEs density:

- The first one for relatively very low UEs density $(U=25)$ and relatively very high UEs density $(U=150)$ where MLB algorithm has low impact on the network performance. Indeed for $U=25$, the heavy load condition (Cond. 1) is very likely to be not verified (i.e. $C A C D_{i}>$ ThPre $_{L B}, \forall i \in C$ ) and the MLB algorithm will very probably not be enabled during the simulation. This explains why the network global throughput for such UEs density is almost the same with and without MLB algorithm activation (Figure 9).

- Whereas for $U=150$, almost all cells are very likely overloaded, then even if a cell $C_{i}$ falls under Cond. 1, HYS $S_{i, j} \quad(j \in S j)$ will remain unchanged (i.e. $\left(C A C D_{j} \leq\right.$ ThAvail $\left.) \Rightarrow\left(\alpha_{i, j}=1\right)\right)$ with and without MLB algorithm activation.

- The second one for $U \in\{50,75,100\}$ where MLB algorithm has significant impact on the network performance since it enhances the network global throughput (Figure 9).

Actually in such case it is very likely that some network cells fall under the heavy load condition (Cond. 1). Also, a heavy loaded cell $C_{i}$ may decrease hysteresis values, $H Y S_{i, j}$ $(j \in S j)$, of neighbouring cells willing to cooperate (i.e. $\left(C A C D_{j}>\right.$ ThAvail $\left.\left._{L B} \Rightarrow 0 \leq \alpha<1\right)\right)$ to enable MLB.

To sum up, the effect of the MLB algorithm and its efficiency on global network throughput are mainly tributary of UEs density and network cells load disparities.

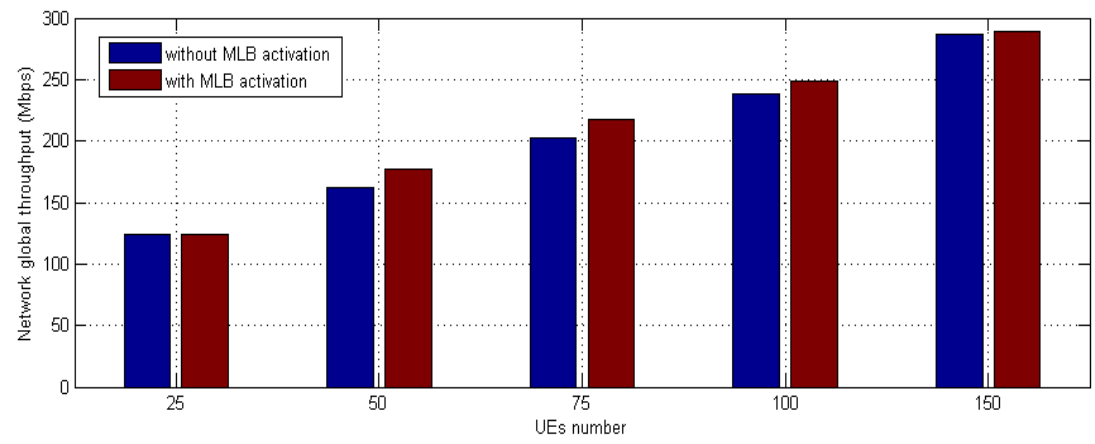

Figure 9. Global network throughput vs UEs density 
Figure 10 highlights a similar behaviour of Jain's fairness index to the global network throughput (Figure 9) versus UEs density. In fact, the Jain's fairness index values are improved when activating the MLB algorithm and this improvement is noticeable except for very low $(U=25)$ and very high $(U=150)$ network UEs densities.

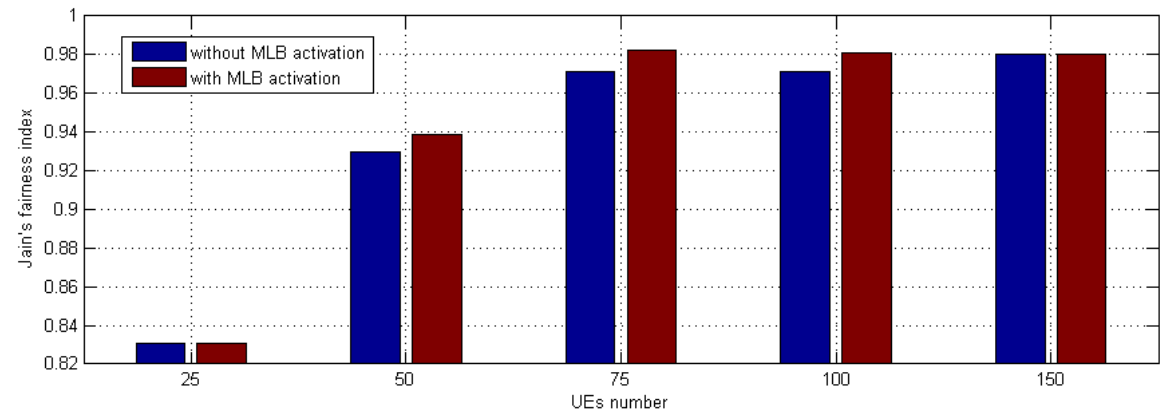

Figure 10. Jain's fairness index vs UEs density

Figure 11 represents the PLR evolution with respect to UEs density with and without activation of the MLB algorithm and shows that PLR is almost the same in both cases except when the total number of UEs in the network is around 100. Three kinds of PLR behaviours with respect to UEs density may be explained as follows:

- The first one corresponds to the case where UEs density is very low $(U=25)$ or very high $(U=150)$. In such case MLB impact is not tangible for the same reasons cited for Figure 9.

- The second one concerns the case where $U \in\{50,75\}$. For such case although the MLB algorithm is very likely to be activated, the PLR remains almost unchanged. This may be explained by the relatively low UEs density not enough high to provoke packet loss.

- The third case corresponds to an UEs density around 100. In this particular case, MLB algorithm activation decreases the PLR value with respect to UEs density. This may be explained by both the relatively high UEs density and the highly probable MLB algorithm enabling.

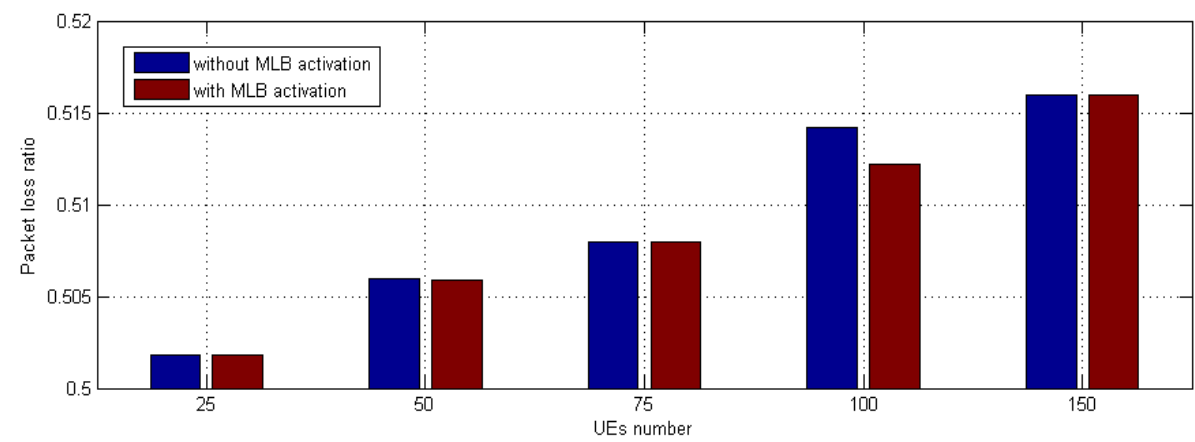

Figure 11. PLR vs UEs density 
Figure 12 illustrates the evolution of the number of successful HOs according to UEs density with and without MLB algorithm activation. The number of successful HOs is almost unchanged when activating MLB algorithm. This may be considered as a significant gain, since a MLB scheme promotes enabling more HOs in order to attenuate load disparity between cells. In conclusion the implemented MLB algorithm seems to be able to find convenient trade-off between different investigated network key performance indicators (KPIs) since it improves global network throughput and PLR without increasing the rate of HO signalling.

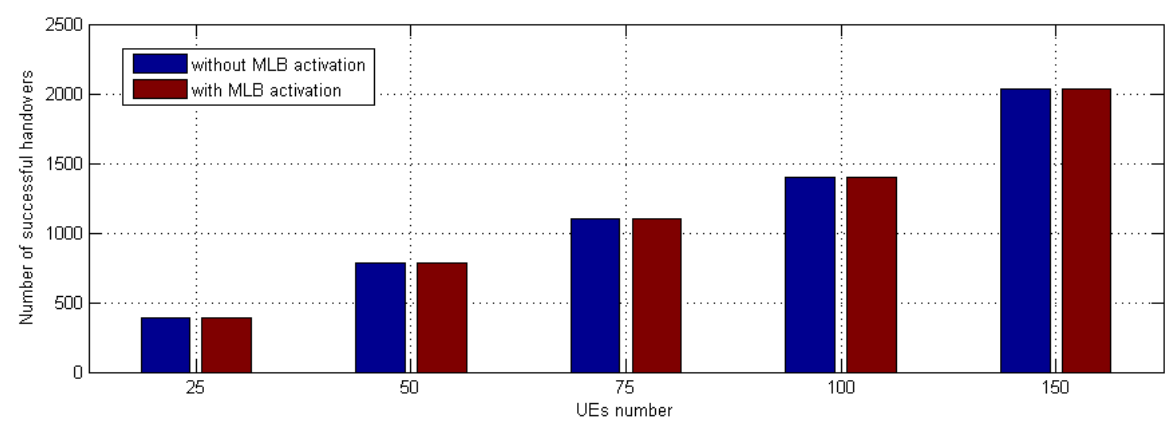

Figure 12. Number of successful HO vs UEs density

\section{ConClusion}

In this paper we propose an implementation, using ns-3, of a MLB algorithm based adaptive HO for downlink LTE SON systems. Since ns-3 with its current version (i.e. ns-3.24) did not implement yet some MM functions, we propose also a basic implementation of Resource Status Reporting EPs which is required for gathering information about network cell's load conditions. Numerical results show that enhancements provided by the proposed MLB algorithm are closely dependent on network UEs density and it's illustrated in this paper through different KPIs. These enhancements concerns particularly PLR reduction, Jain's fairness index and network global throughput increase without HO Overhead. In our future works we intend to further investigate and refine the implemented MM functions. More effort should be made to test and validate the proposed X2AP EPs extensions and to optimize MLB algorithm thresholds.

\section{REFERENCES}

[1] European Telecommunications Standards Institute. LTE; Evolved Universal Terrestrial Radio Access Network (E-UTRAN); Self-configuring and self-optimizing network (SON) use cases and solutions, 2011.

[2] European Telecommunications Standards Institute. LTE; Evolved universal terrestrial radio access network (E-UTRAN); X2 application protocol (X2AP), 2013.

[3] Kyuho Son, Song Chong, and G. Veciana. Dynamic association for load balancing and interference avoidance in multi-cell networks. Wireless Communications, IEEE Transactions on, 8(7):3566-3576, July 2009.

[4] P Mūnoz, R Barco, and I de la Bandera. Load balancing and handover joint optimization in lte networks using fuzzy logic and reinforcement learning. Computer $\quad$ Networks, 76:112-125, 2015.

[5] Wen-Yu Li, Xiang Zhang, Shu-Cong Jia, Xin-Yu Gu, Lin Zhang, Xiao-Yu Duan, and JiaRu Lin. A novel dynamic adjusting algorithm for load balancing and handover co-optimization in lte son. Journal of Computer Science and Technology, 28(3):437-444, 2013. 
[6] Ridha Nasri and Zwi Altman. Handover adaptation for dynamic load balancing in 3gpp long term evolution systems. arXiv preprint arXiv:1307.1212, 2013.

[7] Zhang Peng Huang, Jing Liu, Qiang Shen, Jin Wu, and Xiaoying Gan. A threshold-based multi-traffic load balance mechanism in lte-a networks. In Wireless Communications and Networking Conference (WCNC), 2015 IEEE, pages 1273-1278. IEEE, 2015.

[8] Qi-Ping Yang, Jae-Woo Kim, and Tae-Hyong Kim. Mobility prediction and load balancing based adaptive handovers for lte systems. International Journal on Computer Science and Engineering, 4(4):638, 2012.

[9] European Telecommunications Standards Institute. LTE ; Evolved Universal Terrestrial Radio Access (E-UTRA); Radio Resource Control (RRC); Protocol specification, Nov. 2012.

[10] Lte protocols and specifications. http://lteworld.org/lte-protocols-specifications. Accessed: Dec. 2015.

[11] X2 interface function in lte a connection between two enodebs. http://www.teletopix.org/4g-lte/x2interface-function-in-lte-a-connection-between-two-enodebs/. Accessed: Dec. 2015.

[12] Christopher Cox. An introduction to LTE: LTE, LTE-advanced, SAE and 4G mobile communications. John Wiley \& Sons, 2012.

[13] Alcatel-lucent. The LTE Network Architecture: A comprehensive tutorial, 2013.

[14] Self-organizing networks. http://www.3gpp.org/technologies/keywords-acronyms/105-son. Accessed: Dec. 2015.

[15] Panagiotis Fotiadis, Michele Polignano, Daniela Laselva, Benny Vejlgaard, Preben Mo-gensen, Ralf Irmer, and Neil Scully. Multi-layer mobility load balancing in a heterogeneous lte network. In Vehicular Technology Conference (VTC Fall), 2012 IEEE, pages 1-5. IEEE, 2012.

[16] Dah-Ming Chiu and Raj Jain. Analysis of the increase and decrease algorithms for congestion avoidance in computer networks. Computer Networks and ISDN systems, 17(1):1-14, 1989.

[17] Network simulator 3. url: https://www.nsnam.org/. Accessed: Mai 2015.

[18] Nicola Baldo, Manuel Requena-Esteso, Marco Miozzo, and Raymond Kwan. An open source model for the simulation of LTE handover scenarios and algorithms in ns-3.In Proceedings of the 16th ACM international conference on Modelling, analysis \& simulation of wireless and mobile systems, pages 289-298. ACM, 2013.

[19] Tracy Camp, Jeff Boleng, and Vanessa Davies. A survey of mobility models for ad hocnetwork research. Wireless communications and mobile computing, 2(5):483-502, 2002.

[20] Fan Bai and Ahmed Helmy. A survey of mobility models. Wireless Ad hoc Networks.University of Southern California, USA, 206, 2004.

[21] European Telecommunications Standards Institute. Self-Organizing Networks (SON) Policy Network Resource Model (NRM) Integration Reference Point (IRP); Information Service (IS), 2012.

\section{Authors}

Hana Jouini received the Master's. degree in mathematics and computer science at the university of Paris Descartes, Paris, France in 2013. She is currently a Ph. D. student in computer science and telecommunication at the National engineering school of Tunis and at CNAM Paris.

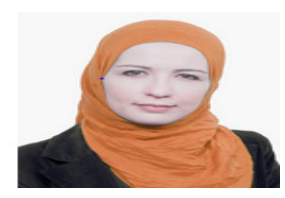

Mohamed Escheikh received in 1992 the Diploma degree in electrical engineering. In 1994, he received a Master's degree and in $2001 \mathrm{a} \mathrm{PhD} \mathrm{degree} \mathrm{with} \mathrm{Distinction} \mathrm{all}$ in electrical engineering from National Engineering School of Tunis ENIT (Tunisia). $\mathrm{He}$ is currently Assistant Professor at ENIT since 2001, member of SYSCOM laboratory at ENIT since 1992 and associate researcher with Vespa Team of Cedric CNAM Paris. His research interests include in particular dependability analysis, queuing systems, Petri nets, mobile networks optimization, cloud computing and power management.

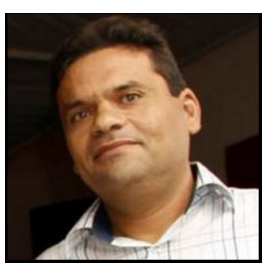


International Journal of Wireless \& Mobile Networks (IJWMN) Vol. 8, No. 4, August 2016

Kamel Barkaoui is full professor at the Department of Computer Science of Conservatoire National des Arts et Métiers (CNAM - Paris) since 2002. He holds a Ph.D in Computer Science (1988) and Habilitation à Diriger des Recherches (1998) from Université Paris 6 (UPMC). His research interests include formal methods for verification, control and performance evaluation of concurrent and distributed systems. He has published over 150 papers on these topics, as well as supervising 25 $\mathrm{PhD}$ theses and several MSc thesis projects. He received the 1995 IEEE Int. Conf. on System Man and Cybernetics Outstanding Paper Award. He served on PCs and as PC

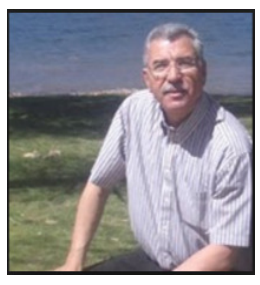
chair and OC chair of a number of international workshops and conferences in his areas of research. He is the SC chair of the International Workshop on Verification and Evaluation of Computer and Communication Systems (VECoS). He was recently general co-Chair of the 18th International Symposium on Formal Methods (2012) and general chair of 35th International Conference on Application and Theory of Petri Nets and Concurrency (Petri Nets 2014) and the 14th International Conference on Application of Concurrency to System Design (ACSD'2014). He is associated editor of the International Journal of Critical Computer-Based Systems (IJCCBS).

Tahar Ezzedine received the M.S degree, the Ph.D degree and the HDR degree (Habilitation à diriger des recherches, "accreditation to supervise research degree) inTelecommunications, all from the National school of engineers of Tunis (ENIT), Tunisia. He is currently with Syscom Research Lab leading an R\&D group for research and development ofwireless networks, wireless sensors network in different fields (environments, agriculture, drought, structure health, ...) enclosing GIS systems for data display his interests also include smart objects and Internet Of Things.

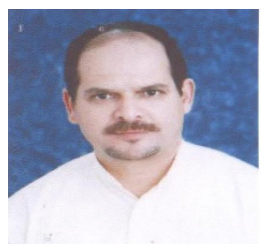

